\title{
Assessment of Operator's Accuracy in Detecting Occlusal Carious Lesion by Visual-Tactile Method in Malaysia
}

ISSN: 2637-7764

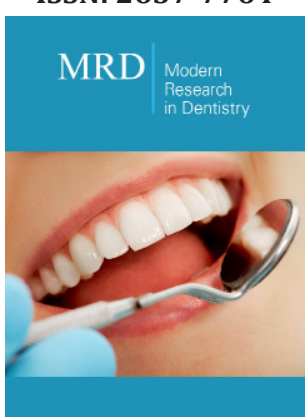

*Corresponding author: Wan Zaripah Wan Bakar, Conservative Dentistry Unit, School of Dental Sciences, Universiti Sains Malaysia Health Campus, 16150 Kubang Kerian, Kelantan, Malaysia,

Email:wzaripah@usm.my

Submission:

Published: 海 October 23, 2019

Volume 4 - Issue 3

How to cite this article: Wan Zaripah Wan B, Min Fui C, Nor Azira Z. Assessment of Operator's Accuracy in Detecting Occlusal Carious Lesion by Visual-Tactile Method in Malaysia. 4(3). MRD.000590.2019.

DOI: 10.31031/MRD.2019.04.000590

Copyright@ Wan Zaripah Wan Bakar, This article is distributed under the terms of the Creative Commons Attribution 4.0 International License, which permits unrestricted use and redistribution provided that the original author and source are credited.

\author{
Wan Zaripah Wan Bakar ${ }^{1 *}$, Min Fui Chong ${ }^{2}$ and Nor Azira Zabidi ${ }^{3}$
}

${ }^{1}$ Conservative Dentistry Unit, School of Dental Sciences, Universiti Sains Malaysia Health Campus, 16150 Kubang Kerian, Kelantan, Malaysia.

${ }^{2}$ Borneo Medical Centre, Lot 10992, Section 64, KTLD, Jalan Tun Jugah, 93350 Kuching, Sarawak, Malaysia.

${ }^{3}$ Ministry of Health, Malaysia.

\begin{abstract}
Visual-tactile method is still a commonly used technique in detecting caries even the International caries detection and Assessment System (ICDAS) has been exposed among dental operators in Malaysia. However, this method has been shown to have high specificity but low sensitivity and reproducibility. This study is conducted to determine the accuracy of visual-tactile method in diagnosing early occlusal caries lesion by dental practitioners in Malaysia. Fourteen posterior teeth were selected as samples for the assessment by 95 respondents from 9 different states which diagnosed each tooth as either sound, early or advanced caries following the criteria set. DIAGNO dent pen which is known as an accurate diagnostic tool in detection occlusal caries was used as a gold standard and the result was compared with the respondent's visual-tactile assessment. Percentage was calculated and the result shown the percentage of accurate early occlusal caries detection using visual-tactile method is $43.23 \%$ (95\% CI: 39.90, 46.56). In addition, the percentage of operators who misdiagnosed advanced lesion as sound or vice versa are $34.74 \%$ (95\% CI: 24.99, 44.49) for 1 misdiagnosis, 6.32\% (95\% CI: 1.33, 11.30) for 2 misdiagnosis and $1.05 \%$ (95\% CI: $-1.03,3.14)$ for 3 mis diagnosis. From this study, the level of accuracy for operators in Malaysia in detecting early occlusal caries lesion using visual-tactile method is relatively low. This finding is hoped to improve clinician awareness of the visual-tactile diagnostic method limitation so they will be more concerned and other devices or systems such as ICDAS should be used.
\end{abstract}

\section{Introduction}

Dental caries, a chronic infectious communicable disease, is one of the most common problems encountered in clinical dentistry [1]. Although caries has affected human population since prehistoric times, the prevalence of this disease has increased greatly in modern worldwide. Statistics shows still a very high incidence of over $70 \%$ of adults in Malaysia according to the 2009 national survey. A thorough understanding of the early lesion morphology is necessary in order to determine the suitable treatment planning and appropriate type of restoration. Correct diagnosis of early caries lesion permit dentists to adopt more conservative management strategies directed at the prevention and cure of dental caries. Current concept of minimally invasive dentistry (MID) concerns about tissue preservation where the philosophies centres around entailing minimal destruction to the tooth structure by removing and replacing with as little tissue loss as possible. It does not suggest that we make small fillings to restore incipient lesion. In this case, correct diagnosis of the lesion is important so that the clinician will give a right treatment.

Various methods have been used in detection of dental caries clinically which includes visual examination method with probing, ICDAS, intraoral radiographs, DIAGNOdent Pen system, fiber optic transillumination (FOTI), digital fiber optic transillumination (DIFOTI) and also Cone-Beam Computed Tomography (CBCT). Golden standard for caries assessment is histological sectioning, and study also found that Micro Computed Tomography (Micro-CT) is the best imaging method for the in vitro measurement of occlusal caries depth [2]. Kouchaji C [3] study also found that laser fluorescence DIAGNOdent pen is a reproducible and accurate 
diagnostic tool that may be very helpful in conjunction with visual examination in the detection of occlusal caries in permanent molars. Occlusal pits and fissures are the most susceptible sites for primary carious lesion which requiring restorative treatment. The pits and fissures provide mechanical shelter for organisms and harbor a community dominated by Streptococci sanguis [1]. Addition to this is the inaccessibility of base of fissure to tooth brush bristles following food impaction. In general, occlusal surface of the tooth can be either sound with or without stained pit and fissure; early caries lesion (discoloration or opacification of fissure) and advanced caries lesion when there is cavitation (EBD Champion Conference, American Dental Association, 2008).

The International Caries Detection and Assessment System (ICDAS) has been introduced which is a clinical scoring system for use in dental education, clinical practice, research and epidemiology. It is designed to lead to better quality information to inform decisions about appropriate diagnosis, prognosis and clinical management at both the individual and public health levels. It also provides a framework to support and facilitate personalized management of the caries process and dental decay for improved long term health outcomes. Deep fissure with staining could be wrongly diagnosed as carious if the operator has a lack of knowledge for accuracy in caries diagnosis. This could lead to surgically restored of the tooth, removing sound tooth structure and is totally unnecessary. In the practice of conservative dentistry, the early caries lesion in enamel will only need preventive methods such as professional fluorides applications or fissure sealants. Remineralisation is recommended rather than restoration. Therefore, definite diagnosis of occlusal caries is crucial and distinguished lesion that can be arrested by non-invasive treatment from those that may justify restorative treatment. Traditional methods of visual examination and probing may need to combine with other diagnostic aids such as radiography, laser or light fluorescence-based methods. Current instruments like micro-CT has a higher sensitivity compared with intraoral image modalities for detection of caries lesions [4]. These modalities also make it possible to measure caries depth, which can be useful in diagnosis, treatment planning and management of dental caries. Thus, it will help with the accurate diagnosis of early occlusal caries lesion. Visual tactile method still remains as the most commonly practiced in caries detection among operators in Malaysia. Not many of them use the other instruments in diagnosing caries especially early caries lesion. This an in vitro prospective study was done to look at the accuracy of this traditional caries detection technique in diagnosing occlusal caries lesion. Once recognized the result of this study will improve clinician awareness of limitation of visual tactile diagnostic method, in turn; help to plan a better way of diagnosing of occlusal caries such as using ICDAS.

\section{Methods and Material}

This in vitro study has been carried out in clinics throughout Malaysia. Fourteen newly extracted human permanent premolars and molars were selected randomly as teeth samples. The criteria including sounds, early caries and minimal cavitated occlusal caries lesion as an advanced lesion sample. Tooth samples was kept in Thymol solution before cleaned and mounted in stone arch, tagged with number 1 to 14 for the later assessment. Ninetyfive respondents which include dentist from the government and private clinics, university dental officers, postgraduate students and specialists and also final year dental students randomly selected from 9 different states were involved. They were requested to assess occlusal surface of each of the 14 samples using visual-tactile method following the guideline given prior to the assessment. Respondents were given dental probe with explanation for only light pressure examination of the occlusal surface allowed. They will then chart the surface for either sound or has early caries or advanced caries lesion.

Later, each tooth sample was examined using laser fluorescence DIAGNOdent LF pen (KaVo, Biberach, Germany) by 2 examiners. Both examiners were trained for standardization to use the equipment following the manufacturer's instructions. Reliability and validity of the device was evaluated and it showed good inter-examiner reproducibility which is $80 \%$. Probe tip 'A' designed for fissure was then tracked across the occlusal fissure and a peak reading recorded and level of caries charted following the DIAGNOdent manufacturer's criteria. Value less than 14 is diagnosed as healthy or sounds; $14-29$ as early caries and 30 and above with cavitation's as advanced caries [5]. Data was collected and analysed using descriptive analysis with SPSS statistic version 22.

\section{Statistical analysis used}

The numerical data collected will be analysed using PASW statistic software version 20.0. All statistical analysis will use independent T- test which will be considered at $\mathrm{p}<0.05$.

\section{Result}

The subject population is 95 dental practitioners from 9 different states in Malaysia with 29 (30.5\%) males and 66 (69.5\%) females. Majority of them, 62 (65.3\%) working with Ministry of Health, Malaysia, 4 (4.2\%) have own a private clinic, 4 (4.2\%) are university lecturers, 11 (11.6\%) are post-graduate students and 14 $(14.7 \%)$ are final year undergraduate students. Among the subjects, $32(33.7 \%)$ of them have less than 1year clinical experience, 26 (27.4\%) have 1 to 5 years of clinical experiences while 37 (38.8\%) of them have more than 5 years clinical experience. A total of 14 occlusal tooth surfaces were examined by each of 95 respondents. For each tooth specifically, the correct diagnosis scoring ranges from 7 (7.4\%) for tooth number 2 and 91 (95.7\%) for tooth number 12 as shown in Table 1. The grand total score for correct diagnosis is 575/1330 (43.23\%) (Table 2). In addition, there are 6 of the teeth involved which have been heavily misdiagnosed; advanced lesion as sound or sound as advanced lesion (Table 3). The results were $34.74 \%$ (95\% CI: 24.99, 44.49) for 1 misdiagnosis, 6.32\% (95\% CI: $1.33,11.30$ ) for 2 misdiagnosis and $1.05 \%$ (95\% CI: $-1.03,3.14)$ for 3 misdiagnosis (Table 4). 
Table 1: Scoring for each tooth.

\begin{tabular}{|c|c|c|c|c|c|}
\hline Tooth & DIAGNOdent Diagnosis & Correct Diagnosis Freq & $\begin{array}{l}\text { Correct Diagno- } \\
\text { sis } \%\end{array}$ & Incorrect Diagnosis Freq & Incorrect Diagnosis \% \\
\hline 1 & Early Caries & 56 & 58.9 & 39 & 41.1 \\
\hline 2 & Early Caries & 7 & 7.4 & 88 & 92.6 \\
\hline 3 & Early Caries & 33 & 34.7 & 62 & 65.3 \\
\hline 4 & Early Caries & 30 & 31.6 & 65 & 68.4 \\
\hline 5 & Early Caries & 32 & 33.7 & 63 & 66.3 \\
\hline 6 & Advance Caries & 48 & 50.5 & 47 & 49.5 \\
\hline 7 & Advance Caries & 27 & 28.4 & 68 & 71.6 \\
\hline 8 & Sound & 39 & 42.1 & 56 & 57.9 \\
\hline 9 & Sound & 78 & 82.1 & 17 & 17.9 \\
\hline 10 & Advance Caries & 52 & 54.7 & 43 & 45.3 \\
\hline 11 & Advance Caries & 57 & 60 & 38 & 40 \\
\hline 12 & Sound & 91 & 95.8 & 4 & 4.2 \\
\hline 13 & Early Caries & 9 & 9.5 & 86 & 90.5 \\
\hline 14 & Early Caries & 15 & 15.8 & 80 & 84.2 \\
\hline
\end{tabular}

Table 2: Grand total score of correct diagnosis.

\begin{tabular}{|c|c|c|c|c|}
\hline Total no of Correct Diagnosis & Percentage (\%) & $\mathbf{9 5 \%}$ CI & Total no of Incorrect Diagnosis & Percentage (\%) \\
\hline $575 / 1330$ & 43.23 & $39.90,46.56$ & $755 / 1330$ & 56.77 \\
\hline
\end{tabular}

Table 3: Percentage of tooth being misdiagnosed advanced lesion as sound or vice versa.

\begin{tabular}{|c|c|c|c|c|c|}
\hline $\begin{array}{c}\text { Caries Level } \\
\text { Tooth } \\
\end{array}$ & Diagnodent Diagnosis & Sound & $\%$ & Advance Caries & $\%$ \\
\hline 6 & Advance Caries & 7 & 7.37 & 48 & 50.53 \\
\hline 7 & Advance Caries & 23 & 24.21 & 27 & 28.42 \\
\hline 8 & Sound & 39 & 41.05 & 7 & 7.37 \\
\hline 9 & Sound & 78 & 82.11 & 1 & 1.05 \\
\hline 10 & Advance Caries & 5 & 5.26 & 52 & 54.74 \\
\hline 11 & Advance Caries & 5 & 5.26 & 57 & 60 \\
\hline 12 & Sound & 91 & 95.79 & 0 & 0 \\
\hline
\end{tabular}

Table 4: Percentage of operators who misdiagnosed advanced lesion as sound or vice versa.

\begin{tabular}{|c|c|c|c|c|}
\hline Category & 1 Misdiagnosis & 2 Misdiagnosis & 3 Misdiagnosis & Total \\
\hline $\mathrm{n}$ & 33 & 2 & 1 & 40 \\
\hline Percentage & $34.74 \%$ & $6.32 \%$ & $1.05 \%$ & 42.11 \\
\hline Percentage $(95 \% \mathrm{CI})$ & $24.99,44.49$ & $1.33,11.30$ & $-1.03,3.14$ & $32.00,52.22$ \\
\hline
\end{tabular}




\section{Discussion}

Accurate diagnosis of the presence or absence of disease is a fundamental requirement in health care. The diagnosis of nonovert occlusal decay is challenging and can be highly subjective, and its inherent uncertainties can lead to widely differing treatment decisions [6]. The problems in caries diagnosis are usually not associated with advanced cavitated large lesions, but primarily involve the early lesions [7]. Therefore, the appropriate means of caries detection and measurement is required as caries prevention is critical especially in developing countries like Malaysia where caries is still a big issue to manage. In our study, fourteen posterior teeth were selected as samples for the assessment, as the occlusal surfaces of molars are especially susceptible to the development of caries due to their anatomical structure, which contains pits and deep fissures [8]. According to Newbrun [9], the caries prevalence rate in industrialized nations has decreased and the occlusal surface of the tooth has become the most affected surface. This showed that despite the reduction in prevalence of dental caries, the reduction is not occurred uniformly for all dental surfaces.

Other opinion by Pinheiro et al. [10] who mention that the occlusal surface is the area that not only most susceptible to dental caries but it is also the most difficult surface for the reliable detection of caries. Dentists have a number of methods for the clinical detection of dental caries on occlusal surface as for this study using visual-tactile and laser fluorescence DIAGNOdent pen. Many operators in this study have difficulty in diagnosing the occlusal caries lesion accurately by using visual-tactile method which shown by the result where the percentage for correct diagnosis is only $43.23 \%$ whereas $56.77 \%$ is incorrect diagnosis. This similar to statement by Lussi [5] who stated that, visual-tactile method is a subjective method and it is difficult to diagnose the occlusal caries using only this method [5]. Caries diagnosis requires a thorough dental examination and close attention to the clinical evidence. Nevertheless, many studies before have demonstrated that diagnosing occlusal caries without cavities is difficult, and that both false-positive and false-negative findings occur frequently [1113]. Correct diagnosis of early caries permit dentists to adopt more conservative management strategies directed at the prevention and cure of dental caries. Anyways, in our study there are 6 teeth involved in being heavily misdiagnosed as advanced lesion when gold standard shows sound; and vice versa. The percentage of operators who misdiagnosed advanced lesion as sound or vice versa are $34.74 \%$ ( 1 misdiagnosis), 6.32\% (2 misdiagnosis) and $1.05 \%$ (3 misdiagnosis) which is quite high. These two contrast diagnosis will lead to 2 different modes of treatment which is preventive (nonsurgical) versus restoration (surgical). The result shown that by relying only on visual-tactile method, it can lead the operators to make a diagnosis mistake and give inappropriate treatment such as surgically restored of sound tooth structure which is unnecessary or underdiagnosed of advanced lesion which was left untreated and can cause further progression of the caries. Therefore, the interpretation of early caries lesions deserves more attention to catch caries at its earliest stages and planning comprehensive dental care, also to avoid unnecessarily and over aggressively "drill and fill" the sound tooth due to misdiagnosis of early carious lesion. The problems in caries diagnosis are usually not associated with advanced lesions, but primarily involve the early lesions (those confined to enamel). It has been suggested that the diagnosis of fissure caries has become more difficult because of the high remineralisation capacity of fluorides tends to mask dentinal caries (Roberta, 1999), thus making it hard to be detected by using visual-tactile method. In vitro studies commonly establish the validity of a detection system by using a "gold standard" against which the diagnostic method can be evaluated.

The most common gold standard used to validate caries detection methods is the histological evaluation of hard tissue sections [14]. However, there are a number of shortcomings in histological methods that we encountered while sectioning the trial sample. Many of the trial samples were fractured during histological sectioning due to improper keeping of the samples prior, the dentine become too hard and increased enamel brittleness. As the laser fluorescence device DIAGNOdent has shown superior results in diagnosing the caries which is almost comparable to histological sectioning [15], then the DIAGNOdent pen was chosen as a gold standard assessment. DIAGNOdent pen is a reproducible and accurate diagnostic tool with sensitivity and specificity of $97 \%$ and $52 \%$ respectively that may be helpful to use in conjunction with visual examination [16]. It is recommended to be used as a complementary tool in order to avoid the occurrence of false-positive and false-negative findings. Furthermore, it is very important to recognize the degree of carious demineralized occlusal surfaces that is susceptible or its probability to respond to remineralizing treatment $[17,18]$.

\section{Conclusion}

Within its limitation, the result from this study showed that the level of accuracy for operators in Malaysia in detecting early occlusal caries lesion using visual-tactile method is relatively low. This preliminary study will hopefully improve clinician's awareness of the limitation of currently used visual-tactile diagnostic method so that they will try other methods, techniques, systems or devices to be used as a supplementary. This is important because definite diagnosis of occlusal caries is crucial as this determines the appropriate treatment planning for the tooth involved with appropriate type of restoration. At the same time, early diagnosing of caries lesion enables remineralization of the lesion, the balance can be tipped in favour of arrestment by diet modification, improving plaque control, and fluoride therapy rather than surgical intervention.

\section{References}

1. Theodore M, Robertson et al. (2002) Sturdevant's Art and science of Operative Dentistry, ( $5^{\text {th }}$ edn), Elsevier Mosby, Riverport Lane, St Louis, Missouri, USA, p. 67.

2. Kamburoğlu K, Kurt H, Kolsuz E, Öztaş B, Tatar I, et al. (2010) Occlusal caries depth measurements obtained by five different imaging modalities. J Digit Imaging 24(5): 804-813. 
3. Kouchaji C (2012) Comparison between a laser fluorescence device and visual examination in the detection of occlusal caries in children. Saudi Dent J 24(3-4): 169-174.

4. Haiter Neto F, Wenzel A, Gotfredsen E (2008) Diagnostic accuracy of cone beam computed tomography scans compared with intraoral image modalities for detection of caries lesions. Dentomaxillofac Radiol 37(1):18-22.

5. Lussi A (1993) Comparison of different methods for the diagnosis of fissure caries without cavitation. Caries Res 27(5): 409-416.

6. McComb D, Tam LE (2001) Diagnosis of occlusal caries: Part I Conventional Methods. J Can Dent Assoc 67(8): 454-457.

7. Basting RT, Serra MC (1999) Occlusal caries: diagnosis and noninvasive treatments. Quintessence Int 30(3): 174-178.

8. Weerheijm, Gruythuysen (1992) Assessment of enamel-dentin caries lesions detection using bitewing PSP digital images. J Appl Oral Sci 19(5): 462-468.

9. Newbrun E (1993) Problems in caries diagnosis. Int Dent J 43(2): 133142.

10. Pinheiro IVA, Medeiros MC, Ferreira MA, Lima KC (2008) Use of laser fluorescence (DIAGNOdent) for in vivo diagnosis of occlusal caries: a systematic review. J. Appl. Oral Sci 12(3).

11. Horowitz AM (2004) A report on the NIH consensus development conference on diagnosis and management of dental caries throughout life. J Dent Res 83: 15-17.

12. Bader JD, Shugars DA, Bonito AJ (2001) Systematic reviews of selected dental caries diagnosis and management methods. J Dent Educ 65(10): 960-968.

13. Sheehy EC, Brailsford SR, Kidd EA, Beighton D, Zoitopoulos L (2001) Comparison between visual examination and a laser fluorescence system for in vivo diagnosis of occlusal caries. Caries Res 35(6): 421-426.

14. Anahita JM, Vitus S (2010) Serial sectioning of teeth and microscopy in cariology research.

15. N Sridhar, S Tandon, Rao N (2009) A comparative evaluation of DIAGNOdent with visual and radiography for detection of occlusal caries: An in vitro study. Indian J Dent Res 20(3): 326-331.

16. Ekstrand KR, Ricketts DN, Kidd EA (1997) Reproducibility and accuracy of three methods for assessment of demineralization depth of the occlusal surface: an in vitro examination. Caries Res 31(3): 224-231.

17. Topping GVA, Pitts NB (2009) International Caries Detection and Assessment System Committee. Clinical visual caries detection, Monogr Oral Sci 21: 15-41.

18. Pitts N (2004) “ICDAS" an international system for caries detection and assessment being developed to facilitate caries epidemiology, research and appropriate clinical management. Community Dent Health 21(3): 193-198. 